\title{
PROPERTIES AND KEPLERIAN ROTATION OF THE HOT CORE IRAS 20126+4104
}

\author{
Jin-Long $\mathrm{Xu}^{1,2,3}$, Jun-Jie Wang ${ }^{1,2}$ and Chang-Chun Ning ${ }^{2,4}$ \\ xuj1@bao.ac.cn
}

Received —

\footnotetext{
${ }^{1}$ National Astronomical Observatories, Chinese Academy of Sciences, Beijing 100012, China

${ }^{2}$ NAOC-TU Joint Center for Astrophysics, Lhasa, Tibet, 850000, China

${ }^{3}$ Graduate University of the Chinese Academy of Sciences, Beijing, 100080, China

${ }^{4}$ Tibet University, Lhasa, Tibet, 850000, China
} 


\begin{abstract}
We present Submillimeter Array observations of the massive star-forming region IRAS 20126+4104 in the millimeter continuum and in several molecular line transitions. With the SMA data, we have detected nine molecular transitions, including DCN, $\mathrm{CH}_{3} \mathrm{OH}, \mathrm{H}_{2} \mathrm{CO}$, and $\mathrm{HC}_{3} \mathrm{~N}$ molecules, and imaged each molecular line. From the $1.3 \mathrm{~mm}$ continuum emission a compact millimeter source is revealed, which is also associated with $\mathrm{H}_{2} \mathrm{O}, \mathrm{OH}$, and $\mathrm{CH}_{3} \mathrm{OH}$ masers. Using a rotation temperature diagram (RTD), we derive that the rotational temperature and the column density of $\mathrm{CH}_{3} \mathrm{OH}$ are $200 \mathrm{~K}$ and $3.7 \times 10^{17} \mathrm{~cm}^{-2}$, respectively. The calculated results and analysis further indicate that a hot core coincides with IRAS 20126+4104. The position-velocity diagrams of $\mathrm{H}_{2} \mathrm{CO}\left(3_{0,3}-2_{0,2}\right)$ and $\mathrm{HC}_{3} \mathrm{~N}$ (25-24) clearly present Keplerian rotation. Moreover, $\mathrm{H}_{2} \mathrm{CO}\left(3_{0,3}-2_{0,2}\right)$ is found to trace the disk rotation for the first time.
\end{abstract}

Subject headings: ISM: individual (IRAS 20126+4104) — ISM: kinematics and dynamics — ISM: molecules — stars: formation 


\section{Introduction}

Massive star formation, especially in the earliest phase, is poorly understood, due to the large distances involved ( $\geq 1 \mathrm{kpc}$ ), complex cluster formation environments and shorter evolutionary timescales of massive stars. Hot cores represent the early phase in the process of massive star-formation (Qin et al. 2010). The hot cores are defined as compact ( $\leq$ $\left.0.1 \mathrm{pc}, \mathrm{n} \geq 10^{7} \mathrm{~cm}^{-3}\right)$, relatively high temperature $\left(T_{\mathrm{k}} \geq 100 \mathrm{~K}\right)$ cloud cores (Kurtz et al. 2000). Thus, observation of hot cores with high angular resolution and various molecular lines can provide important information on the early evolutionary phase of massive star formation.

IRAS 20126+4104, at a distance of $1.7 \mathrm{kpc}$ from the Sun (Dame \& Thaddeus, 1985; Wilking et al. 1989; Moscadelli et al. 2011), is a massive star-forming region with a bolometric luminosity of $1.3 \times 10^{4} L_{\odot}$. Previous observations of $\mathrm{NH}_{3}, \mathrm{CH}_{3} \mathrm{CN}$ and $\mathrm{CS}$ molecular lines toward IRAS 20126+4104 indicated that a Keplerian disk was detected around $\mathrm{a} \sim 7-24 M_{\odot}$ point mass (Zhang et al. 1998; Keto \& Zhang 2010; Cesaroni et al. 1999, 2005). Traced in $\mathrm{CO}, \mathrm{NH}_{3}, \mathrm{SiO}, \mathrm{HCO}^{+}, \mathrm{CH}_{3} \mathrm{OH}$ and $\mathrm{H}_{2}$ molecular lines (Shinnaga, et al. 2008; Su et al. 2007; Zhang et al. 1999; Cesaroni et al. 1997, 1999, 2005; Shepherd et al. 2000), a bipolar jet/outflow system was widely investigated toward IRAS 20126+4104. In addition, $\mathrm{H}_{2} \mathrm{O}, \mathrm{OH}$ and $\mathrm{CH}_{3} \mathrm{OH}$ masers in IRAS $20126+4104$ were revealed from observations at centimeter bands (Tofani et al. 1995; Trinidad et al. 2005; Minier et al. 2001; Edris et al. 2005). The $\mathrm{CH}_{3} \mathrm{OH}$ masers might be associated with a hot core (Minier et al. 2001). From VLA observations toward IRAS 20126+4104, Tofani et al. (1995) did not detect the 1.3 and $3.6 \mathrm{~cm}$ continuum emission, but Hofner et al. (1999) detected two elongated structures at the $3.6 \mathrm{~cm}$ continuum band. They concluded that the emission was associated with thermal ionized jets and not from a UCHıI region. Moreover, IRAS 20126+4104 has the typical colors of UCHıI (Wood \& Churchell 1989), suggesting that 
IRAS 20126+4104 may be a precursor of a UCHII region (Molinari et al. 1996). Thus, IRAS $20126+4104$ provides us with an opportunity to study the early evolution of massive star formation.

We have carried out multiline observations toward the massive star-forming region IRAS 20126+4104 with the Submillimeter Array. Various molecular lines are used to investigate the physical and chemical processes of IRAS 20126+4104. So far, only a few molecular lines exclusively indicate rotational motions (Beuther 2007). In this paper, we find new molecular line $\left(\mathrm{H}_{2} \mathrm{CO}\right)$ which can trace the Keplerian disk. In Sect. 2, we summarize the observations. In Sect. 3, we give the general results. We discuss core properties and core kinematics in Sect. 4.1 and Sect. 4.2, respectively. In Sect. 5, we summarize our main conclusions.

\section{OBSERVATIONS AND DATA REDUCTION}

The data are from SMA archive. Observations toward IRAS 20126+4104 were carried out with the SMA on 2004 August 23, at 218 (lower sideband) and $228 \mathrm{GHz}$ (upper sideband). The typical system temperature was $130 \mathrm{~K}$. The compact configuration with seven antennas was used. The phase center was R.A(J2000.0) $=20^{\mathrm{h}} 14^{\mathrm{m}} 26^{\mathrm{s}} .00$ and decl. $(\mathrm{J} 2000.0)=41^{\circ} 13^{\prime} 31^{\prime \prime} .50$. The spectral resolution is $0.8125 \mathrm{MHz}$, corresponding to a velocity resolution of $1.1 \mathrm{~km} \mathrm{~s}^{-1}$. The absolute flux density scales were determined from observations of Uranus, and the bright quasar 3C 279 was used for bandpass calibration. QSO 2015+371 and QSO 2202+422 were observed for the antenna gain corrections. The calibration and imaging were performed in Miriad. The continuum map was made using the line-free channels. The spectral cubes were constructed using the continuum-subtracted spectral channels. Self-calibration was performed to the continuum data. The gain solutions from the continuum were applied to the line data. The synthesized beam size of continuum 
and line images with robust weighting was approximately $1^{\prime \prime} .26 \times 0^{\prime \prime} .96\left(\right.$ P.A. $\left.=89^{\circ} .0\right)$.

\section{RESULTS}

\subsection{Continuum Emission at $1.3 \mathrm{~mm}$}

Figure 1 is the $1.3 \mathrm{~mm}$ continuum map of IRAS $20126+4104$ obtained by combining the lower and upper sideband data. The continuum emission shows a compact source, which is unresolved in the beam at $1.3 \mathrm{~mm}$. By using a two-dimensional Gaussian fit for the continuum emission, we obtained that the total flux density is $0.62 \pm 0.03 \mathrm{Jy}$, the deconvolved source size is $0.8^{\prime \prime} \times 0.5^{\prime \prime}\left(\right.$ P.A. $\left.=87.9^{\circ}\right)$, and the peak position is R.A.(J2000) $=20^{\mathrm{h}} 14^{\mathrm{m}} 26 .{ }^{\mathrm{s}} 040\left(\Delta\right.$ R.A. $\left.= \pm 0.04^{\prime \prime}\right)$, decl. $(\mathrm{J} 2000)=+41^{\circ} 13^{\prime} 32 .^{\prime \prime} 55\left(\Delta\right.$ decl. $\left.= \pm 0.03^{\prime \prime}\right)$ with an intensity of $0.46 \pm 0.05 \mathrm{Jy}$ beam $^{-1}$. The peak position of the $3.3 \mathrm{~mm}$ continuum emission is R.A.(J2000) $=20^{\mathrm{h}} 14^{\mathrm{m}} 26 .^{\mathrm{s}} 008\left(\Delta\right.$ R.A. $\left.= \pm 0.01^{\prime \prime}\right)$, decl. $(\mathrm{J} 2000)=+41^{\circ} 13^{\prime} 32 .^{\prime \prime} 73$ $\left(\Delta\right.$ decl. $\left.= \pm 0.01^{\prime \prime}\right)$ (Cesaroni et al. 1997). For the $7 \mathrm{~mm}$ continuum emission (Hofner et al. 1999), the peak position is R.A.(J2000) $=20^{\mathrm{h}} 14^{\mathrm{m}} 26 .^{\mathrm{s}} 026$, decl.(J2000) $=+41^{\circ} 13^{\prime} 32 .^{\prime \prime} 70$. The three positions coincide within the uncertainties. $\mathrm{H}_{2} \mathrm{O}, \mathrm{OH}$ and $\mathrm{CH}_{3} \mathrm{OH}$ masers in IRAS 20126+4104 have been detected from observations at centimeter wavelengths (Tofani et al. 1995; Trinidad et al. 2005; Minier et al. 2001; Edris et al. 2005). In Figure 1, $\mathrm{H}_{2} \mathrm{O}$ masers are shown with the blue filled triangles. The green open squares present $\mathrm{OH}$ masers. The filled black circles show $\mathrm{CH}_{3} \mathrm{OH}$ masers. Most of the masers are associated with the $1.3 \mathrm{~mm}$ continuum emission, but are not located at the peak position of the 1.3 continuum emission. 


\subsection{Molecular Line Emission}

In the frequency coverage of the $2 \mathrm{GHz}$ band, deuterated hydrogen cyanide (DCN), formaldehyde $\left(\mathrm{H}_{2} \mathrm{CO}\right)$, cyanoacetylene $\left(\mathrm{HC}_{3} \mathrm{~N}\right)$ and methanol $\left(\mathrm{CH}_{3} \mathrm{OH}\right)$ emission lines were identified by use of the CDMS and JPL catalog ( Müller et al. 2005; Pickett et al. 1998), as done by other authors in the same frequency range toward Orion (Sutton et al. 1985) and Sgr B2 (Qin et al. 2008). The transition, rest frequency and upper level energy $\left(E_{\mathrm{u}}\right)$ of each molecular line are presented in Table 1.

Molecular line images can provide valuable information on the spatial distribution of molecules and kinematics, then we imaged the molecular lines of $\mathrm{DCN}, \mathrm{CH}_{3} \mathrm{OH}, \mathrm{H}_{2} \mathrm{CO}$, and $\mathrm{HC}_{3} \mathrm{~N}$. The $1.3 \mathrm{~mm}$ continuum emission has been subtracted before computing these maps. Figure 2 presents the integrated intensity maps of each molecular line. " $\times$ " indicates the peak position of the continuum emission. In Figure 2, each line emission is spatially coincident with the continuum emission.

Spectra of molecular emission are present in Figure 3. The spectra were extracted from the peak positions of each integrated intensity map. We have made Gaussian fits

to all the spectra. The central line velocity $\left(V_{\mathrm{LSR}}\right)$, peak intensity $\left(I_{\mathrm{p}}\right)$, and full width at half-maximum $(\Delta V)$ are summarized in Table 1.

\section{DISCUSSION}

\subsection{Core Properties}

Our interferometric continuum and multiline observations toward the massive starforming region IRAS $20126+4104$ reveal a compact millimeter source, which is associated with $\mathrm{H}_{2} \mathrm{O}, \mathrm{OH}$, and $\mathrm{CH}_{3} \mathrm{OH}$ masers. Millimeter continuum emission mainly traces warm 
dust heated by the embedded young stellar object. Four transitions of $\mathrm{CH}_{3} \mathrm{OH}$ have been detected in IRAS 20126+4104, so we can use a rotation temperature diagram (RTD) to estimate the rotation temperature and the column density. Assuming local thermodynamic equilibrium (LTE), lines being optically thin and gas emission filling the beam, the rotation temperature and the beam-averaged column density can be expressed by (Goldsmith \& Langer 1999; Liu et al. 2002; Qin et al. 2010)

$$
\ln \left(\frac{N_{u}}{g_{u}}\right)=\ln \left(\frac{N_{T}}{Q_{\text {rot }}}\right)-\frac{E_{u}}{T_{\text {rot }}},
$$

Where $N_{u}$ is the column density of the upper energy level, $g_{u}$ is the degeneracy factor in the upper energy level, $N_{T}$ is the total beam-averaged column density, $Q_{\text {rot }}$ is the rotational partition function, $E_{u}$ is the upper level energy in $\mathrm{K}$, and $T_{r o t}$ is the rotation temperature. Figure 4 is the rotation temperature diagram. A linear least-squares fit is performed toward the four transitions of $\mathrm{CH}_{3} \mathrm{OH}$. The RTD can be corrected by multiplying $N_{u} / g_{u}$ by the optical depth correction factor $C_{\tau}=\tau /\left(1-e^{\tau}\right)$, where $\tau$ is the optical depths. According to Qin et el. (2010), we iteratively applied the $C_{\tau}$ correction to the RTD until the solution converged. After the optical depth is corrected, the rotation temperature and the beam-averaged column density are $200 \pm 57 \mathrm{~K}$ and $(3.7 \pm 1.3) \times 10^{17} \mathrm{~cm}^{-2}$, respectively. The rotation temperature of $200 \pm 57 \mathrm{~K}$ is the same as that estimated in the $\mathrm{CH}_{3} \mathrm{OH}(2-1)$ and (5-4) lines (171 K; Cesaroni et al. 2005), and in the $\mathrm{CH}_{3} \mathrm{CN}$ lines (201 K ; Cesaroni et al. 1997).

In the Orion molecular cloud, the strongest $\mathrm{CH}_{3} \mathrm{OH}$ emission is from the compact ridge (Schilke et al. 1997), giving fractional abundances relative to $\mathrm{H}_{2}$ of $1.2 \times 10^{-7}$. We adopt $0.007 \mathrm{pc}$ as the deconvolved size of the $\mathrm{CH}_{3} \mathrm{OH}$ emission derived from the integrated intensity maps of $\mathrm{CH}_{3} \mathrm{OH}$, which is smaller than the canonical size of $0.1 \mathrm{pc}$ that is commonly quoted for hot molecular cores (Kurtz et al. 2000). By using the fractional abundance in the Orion molecular cloud, the calculated $\mathrm{H}_{2}$ density is $4 \times 10^{7} \mathrm{~cm}^{-3}$. The 
relatively higher gas temperature $(200 \pm 57 \mathrm{~K}), \mathrm{H}_{2}$ density $\left(10^{7} \mathrm{~cm}^{-3}\right)$, and smaller size $(0.007 \mathrm{pc})$ indicate a hot core in this region. Following Gibb et al. (2000), we adopt 3 $\times 10^{24} \mathrm{~cm}^{-2}$ as a typical $\mathrm{H}_{2}$ column density. We obtain that the fractional abundance of $\mathrm{CH}_{3} \mathrm{OH}$ relative to $\mathrm{H}_{2}$ is $8 \times 10^{-6}$. The fractional abundance of $\mathrm{CH}_{3} \mathrm{OH}$ is close to that in the Orion hot core (Blake et al. 1987), but higher than that in the Sgr B2 core (Qin et al. 2008).

\subsection{Core Kinematics}

The different characteristics of molecular lines may be used as tools to trace various physical processes of massive star formation. To understand the kinematic signatures of the rotating disk, we use the $\mathrm{H}_{2} \mathrm{CO}\left(3_{0,3}-2_{0,2}\right)$ and $\mathrm{HC}_{3} \mathrm{~N}(25-24)$ lines to construct a position-velocity diagram cut through the continuum peak position at a position angle of $58^{\circ}$. In Figure 5, the continuum emission is redshifted to the southwest and blueshifted to the northeast. The position-velocity diagrams of $\mathrm{H}_{2} \mathrm{CO}\left(3_{0,3}-2_{0,2}\right)$ and $\mathrm{HC}_{3} \mathrm{~N}(25-24)$ clearly present Keplerian rotation. The Keplerian rotation traced in the $\mathrm{HC}_{3} \mathrm{~N}(25-24)$ line is extremely similar to the model disk constructed by Zhang et al. (1998). So far, for many sources only a few molecular lines exclusively indicated rotating motions in massive star formation (Beuther 2007), e.g., $\mathrm{CH}_{3} \mathrm{CN}, \mathrm{C}^{34} \mathrm{~S}, \mathrm{NH}_{3}, \mathrm{HCOOCH}_{3}, \mathrm{C}^{17} \mathrm{O}, \mathrm{H}_{2}^{18} \mathrm{O}, \mathrm{HN}_{13} \mathrm{C}$, $\mathrm{HC}_{3} \mathrm{~N}$ and $\mathrm{CH}_{3} \mathrm{OH}$. Here we find a new molecule $\left(\mathrm{H}_{2} \mathrm{CO} 3_{0,3}-2_{0,2}\right)$, which can be used to investigate the rotational signatures of a disk.

In Figure 5, a velocity gradient in the NE-SW direction is clearly depicted. The velocities of the two emission peaks are -6.0 and $-2.0 \mathrm{~km} \mathrm{~s}^{-1}$ with $0.60^{\prime \prime}$ separation for the $\mathrm{H}_{2} \mathrm{CO}\left(3_{0,3}-2_{0,2}\right)$ molecule, and -5.6 and $-3.0 \mathrm{~km} \mathrm{~s}^{-1}$ with $0.55^{\prime \prime}$ separation for the $\mathrm{HC}_{3} \mathrm{~N}$ (25-24) molecule, respectively. Assuming equilibrium between the rotational and 
gravitational force at the outer radius of the Keplerian disk:

$$
M_{\mathrm{rot}}=\frac{V^{2} r}{G}=2.3 \times 10^{2} V^{2}\left(\mathrm{~km} \mathrm{~s}^{-1}\right) r(\mathrm{pc}) M_{\odot},
$$

where $V$ is the velocity difference, $r$ is the spatial separation of the emission peaks and $G$ is the gravitational constant. The derived dynamical mass is $18.0 M_{\odot}$ and $7.1 M_{\odot}$ in the $\mathrm{H}_{2} \mathrm{CO}\left(3_{0,3}-2_{0,2}\right)$ and $\mathrm{HC}_{3} \mathrm{~N}(25-24)$ molecular lines, respectively. The calculated dynamical mass is smaller than a mass of $\sim 20 M_{\odot}$ obtained by Zhang et al (1998) in the $\mathrm{NH}_{3}$ molecular lines.

\section{SUMMARY}

We have observed the massive star-forming region IRAS $20126+4104$ in the millimeter continuum and in several molecular line transitions. The $1.3 \mathrm{~mm}$ continuum emission reveals a compact millimeter source which is associated with $\mathrm{H}_{2} \mathrm{O}, \mathrm{OH}$, and $\mathrm{CH}_{3} \mathrm{OH}$ masers. From the SMA data, nine molecular transitions, including $\mathrm{DCN}, \mathrm{CH}_{3} \mathrm{OH}, \mathrm{H}_{2} \mathrm{CO}$, and $\mathrm{HC}_{3} \mathrm{~N}$ molecules are detected. The rotational temperature and the column density of $\mathrm{CH}_{3} \mathrm{OH}$ emission are $200 \mathrm{~K}$ and $3.7 \times 10^{17} \mathrm{~cm}^{-2}$, respectively. The calculated results and analysis further indicate that a hot core coincides with IRAS 20126+4104. A Keplerian rotational disk in the IRAS $20126+4104$ hot core is verified in the $\mathrm{H}_{2} \mathrm{CO}\left(3_{0,3}-2_{0,2}\right)$ and $\mathrm{HC}_{3} \mathrm{~N}(25-24)$ lines. $\mathrm{H}_{2} \mathrm{CO}\left(3_{0,3}-2_{0,2}\right)$ is found to trace the disk rotation for the first time. The dynamical mass of the Keplerian rotational disk is $18.0 M_{\odot}$ and $7.1 M_{\odot}$, determined in the $\mathrm{H}_{2} \mathrm{CO}$ $\left(3_{0,3}-2_{0,2}\right)$ and $\mathrm{HC}_{3} \mathrm{~N}(25-24)$ molecular lines, respectively.

We thank the SMA staff for the observations and the anonymous referee whose insights greatly improved this manuscript. We also thank Dr. H. Shi and other people for providing help with the data reduction and analysis. This work was supported by the National Natural Science Foundation of China under Grant No.10473014. 


\section{REFERENCES}

Beuther, H. 2007, in IAU Symp. 237, Triggered Star Formation in a Turbulent Interstellar Medium, ed. B. G. Elmegreen \& J. Palouš (Cambridge: Cambridge Univ. Press), 148

Blake, G. A., Sutton, E. C., Masson, C. R., \& Phillips, T. G. 1987, ApJ, 315, 621

Cesaroni R., Felli M., Walmsley C. M., Olmi L. 1997, A\&A, 325, 725

Cesaroni R., Felli M., Jenness T., Neri R., Olmi L., Robberto M., Testi L., Walmsley C. M. 1999, A\&A, 345, 949

Cesaroni R., Neri R., Olmi L., Testi L., Walmsley C. M., Hofner P. 2005, A\&A, 434, 1039

Dame, T. M., \& Thaddeus, P. 1985, ApJ, 297, 751

Edris, K. A., Fuller, G. A., Cohen, R. J., \& Etoka, S. 2005, A\&A, 434, 213

Gibb, E., Nummelin, A., Irvine, W. M., Whittet, D. C. B., \& Bergman, P. 2000, ApJ, 545, 309

Goldsmith, P. F., \& Langer, W. D. 1999, ApJ, 517, 209

Hofner, P., Cesaroni, R., Rodríguez, L. F., \& Martí, J. 1999, A\&A, 345, L43

Keto, E., \& Zhang, Q. 2010, MNRAS, 406, 102

Kurtz, S., et al. 2000, in Protostars and Planets IV, ed. V. Mannings, A. Boss, \& S. Russell (Tucson: Univ. Arizona Press), 299

Liu, S.-Y., Girart, J. M., Remijan, A., \& Snyder, L. E. 2002, ApJ, 576, 255

Minier, V., Conway, J. E, \& Booth, R. S. 2001, ApJ, 369, 278

Molinari, S., Brand, J., Cesaroni, R., \& Palla, F. 1996, A\&A, 308, 573 
Moscadelli, L., Cesaroni, R., Rioja, M. J., Dodson, R., \& Reid, M. J. 2011, A\&A, 526, 66

Müller, H. S. P., Schlöder, F., Stutzki, J., \& Winnewisser, G. 2005, J. Mol. Struct., 742, 215

Pickett, H. M., Poynter, R. L., Cohen, E. A., Delitsky, M. L., Pearson, J. C., \& Müller, H. S. P. 1998, J. Quant. Spectrosc. Radiat. Transfer, 60, 883

Qin, S.-L., Zhao, J.-H., Moran, J. M., et al. 2008, ApJ, 677, 353

Qin, S.-L., Wu, Y. F., Huang, M. H., Zhao, G., Li, D., Wang, J.-J., Chen, S. 2010, ApJ, 711,399

Schilke, P., Groesbeck, T. D., Blake, G. A., \& Phillips, T. G. 1997, ApJS, 108, 301

Shepherd, D. S., Yu, K. C., Bally, J., \& Testi, L. 2000, ApJ, 535, 833

Shinnaga, H., Phillips, T. G., Furuya, R. S., et al. 2008, ApJ, 682, 1103

Su, Y.-N., Liu, S. Y., Chen, H. R., et al. 2007, ApJ, 671, 571

Sutton, E. C., Blake, G. A., Masson, C. R., Phillips, T. G., 1985, ApJS, 58, 341

Trinidad, M. A., Curiel, S., Migenes, V., et al. 2005, ApJ, 130, 2206

Tofani, G., Felli, M., Taylor, G. B., \& Huang, T. R. 1995, A\&AS, 112, 299

Wilking, B. A., Blackwell, J. H., Mundy, L. G., Howe, J. E. 1989, ApJ, 345, 257

Wood, D. O. S., \& Churchwell, E. 1989, ApJ, 340, 265

Zhang, Q., Hunter, T. R., \& Sridharan, T. K. 1998, ApJ, 505, L151

Zhang, Q., Hunter, T. R., Sridharan, T. K., \& Cesaroni, R. 1999, ApJ, 527, L117

This manuscript was prepared with the AAS IATEX macros v5.2. 


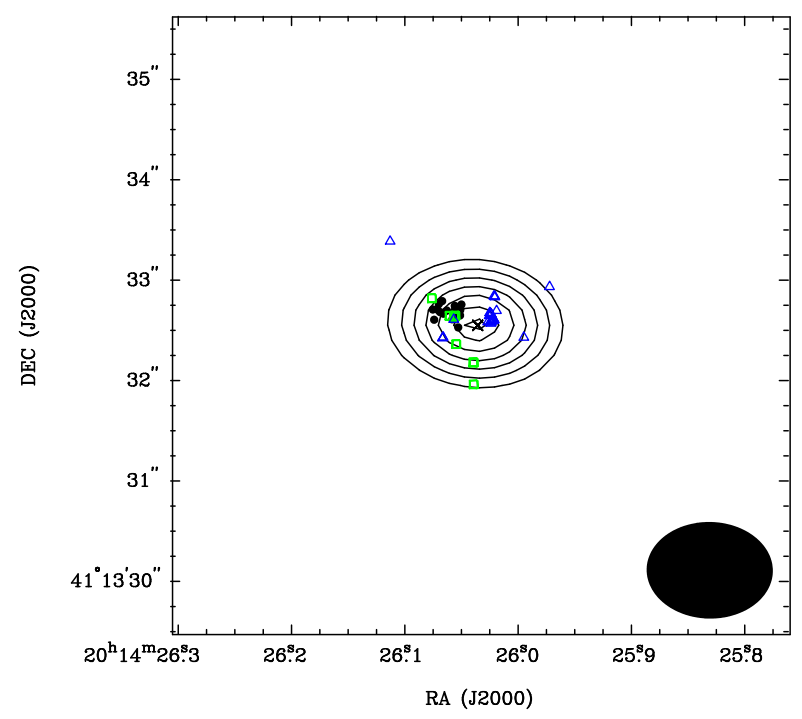

Fig. 1.- Continuum map toward IRAS $20126+4104$ at $1.3 \mathrm{~mm}$ obtained by combining the data from both sidebands (218 and $228 \mathrm{GHz}$ ). The contours are at $-3,3,4,5,6,7,8$, and $9 \sigma$. The rms noise level is $0.05 \mathrm{Jy}$ beam $^{-1}(1 \sigma)$. The synthesized beam is $1.3^{\prime \prime} \times 0.8^{\prime \prime}, \mathrm{P} . \mathrm{A} .=88.9^{\circ}$ (lower right corner). " $\times$ " indicates the peak position of the continuum from the Gaussian fit. $\mathrm{H}_{2} \mathrm{O}$ masers are shown with blue filled triangles. The green open squares present $\mathrm{OH}$ masers. The filled black circles show $\mathrm{CH}_{3} \mathrm{OH}$ masers. 


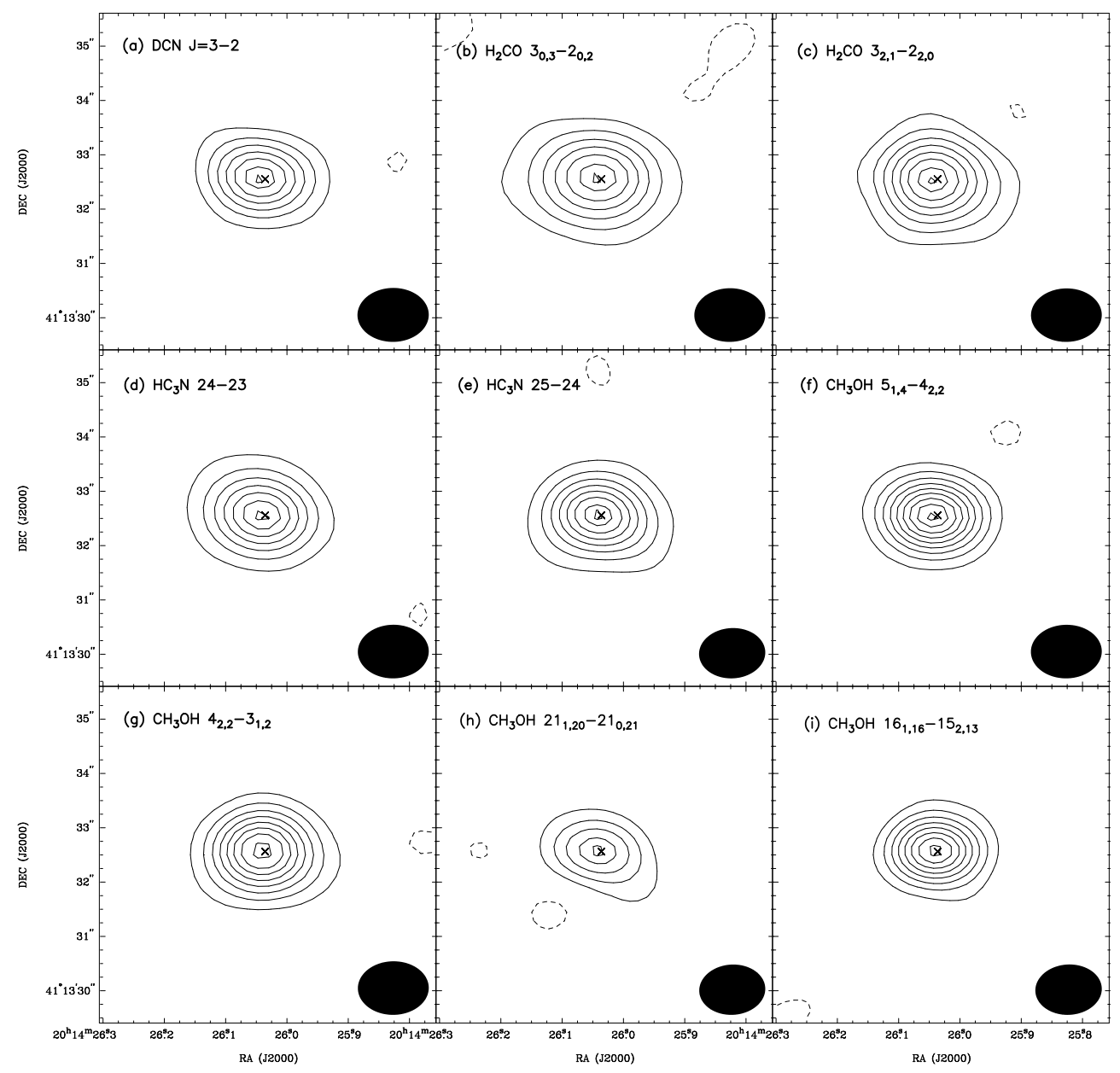

Fig. 2.- (a) The DCN 3-2 integrated intensity map. The contour levels are at -2.2, 2.2, 4.4, 6.6, 8.8, 11.0, 13.2 and $14.3 \mathrm{Jy}_{\text {beam }}{ }^{-1} \mathrm{~km} \mathrm{~s}^{-1}$. (b) The $\mathrm{H}_{2} \mathrm{CO} 3_{0,3}-2_{0,2}$ integrated intensity map. The contour levels are at -1.0, 2.5, 5.0, 7.5, 9.0, 12.5, 15.0 and $16.2 \mathrm{Jy}_{\mathrm{beam}}^{-1} \mathrm{~km} \mathrm{~s}^{-1}$. (c) The $\mathrm{H}_{2} \mathrm{CO} 3_{2,1}-2_{2,0}$ integrated intensity map. The contour levels are at -1.5, 2.4, 4.8, 7.2, 9.6, 12.0, 14.4, 16.8 and 18.1 $\mathrm{Jy}_{\text {beam }}{ }^{-1} \mathrm{~km} \mathrm{~s}^{-1}$. (d) The $\mathrm{HC}_{3} \mathrm{~N} 24-23$ integrated intensity map. The contour levels are at $-1.5,2.3,4.5,6.7,8.9,11.1,13.3$ and $15.1 \mathrm{Jy} \mathrm{beam}^{-1} \mathrm{~km}$ $\mathrm{s}^{-1}$. (e) The $\mathrm{HC}_{3} \mathrm{~N} 25-24$ integrated intensity map. The contour levels are at -1.6, 2.1, 4.2, 6.3, 8.4, 10.5, 12.6, 14.7 and $15.9 \mathrm{Jy} \mathrm{beam}^{-1} \mathrm{~km} \mathrm{~s}^{-1}$. (f) The $\mathrm{CH}_{3} \mathrm{OH} 5_{1,4}{ }_{4}{ }_{2,2}$ integrated intensity map. The contour levels are at -1.1, 2.2, 4.4, 6.6, 8.8, 11.0, 13.2, 15.4, 17.6, and 19.3 Jy beam ${ }^{-1} \mathrm{~km} \mathrm{~s}^{-1}$. (g) The $\mathrm{CH}_{3} \mathrm{OH} 5_{1,4}-4_{2,2}$ integrated intensity map. The contour levels are at $-2.1,2.8,5.6,8.4,11.2,14.0,16.8,19.6$ and $22.8 \mathrm{Jy} \mathrm{beam}^{-1} \mathrm{~km} \mathrm{~s}^{-1}$. (h) The $\mathrm{CH}_{3} \mathrm{OH} 21_{1,20}-21_{0,21}$ integrated intensity map. The contour levels are at -1.4, 1.8, 3.6, 5.4, 7.2, and $8.2 \mathrm{Jy} \mathrm{beam}^{-1} \mathrm{~km} \mathrm{~s}^{-1}$. (i) The $\mathrm{CH}_{3} \mathrm{OH} 16_{1,16}-15_{2,13}$ integrated intensity map. The contour levels are at $-1.5,2.0,4.1,6.1,8.2,10.2,12.2,14.3$ and $16.1 \mathrm{Jy} \mathrm{beam}^{-1} \mathrm{~km} \mathrm{~s}^{-1}$. " $\times$ " 
Table 1: Observed Parameters of Each Line

\begin{tabular}{|c|c|c|c|c|c|c|c|}
\hline Molecule & Transition & $\begin{array}{c}\text { Rest Frequency } \\
(\mathrm{GHz})\end{array}$ & $\begin{array}{l}E_{\mathrm{u}} \\
(\mathrm{K})\end{array}$ & $\begin{array}{c}V_{\mathrm{LSR}} \\
\left(\mathrm{km} \mathrm{s}^{-1}\right)\end{array}$ & $\begin{array}{c}I_{\mathrm{p}} \\
\left(\mathrm{Jy} \mathrm{beam}^{-1}\right)\end{array}$ & $\begin{array}{c}\Delta V \\
\left(\mathrm{~km} \mathrm{~s}^{-1}\right)\end{array}$ & $\begin{array}{l}\text { Channel rms } \\
\left(\mathrm{Jy} \mathrm{beam}^{-1}\right)\end{array}$ \\
\hline $\mathrm{DCN}$ & $3-2$ & 217.239 & 20.9 & $-3.4(0.2)$ & $1.65(0.08)$ & $8.0(0.4)$ & 0.2 \\
\hline \multirow[t]{2}{*}{$\mathrm{H}_{2} \mathrm{CO}$} & $3_{0,3}-2_{0,2}$ & 218.222 & 21.0 & $-3.8(0.3)$ & $2.38(0.19)$ & $8.6(0.8)$ & 0.3 \\
\hline & $3_{2,1}-2_{2,0}$ & 218.760 & 68.1 & $-5.1(0.3)$ & $2.09(0.13)$ & $7.5(0.6)$ & 0.2 \\
\hline \multirow[t]{2}{*}{$\mathrm{HC}_{3} \mathrm{~N}$} & $24-23$ & 218.325 & 131.0 & $-4.1(0.2)$ & $1.78(0.11)$ & $7.8(0.6)$ & 0.2 \\
\hline & $25-24$ & 227.419 & 141.9 & $-4.6(0.1)$ & $2.53(0.09)$ & $6.7(0.3)$ & 0.2 \\
\hline \multirow[t]{4}{*}{$\mathrm{CH}_{3} \mathrm{OH}$} & $5_{1,4}-4_{2,2}$ & 216.946 & 55.9 & $-3.8(0.2)$ & $2.02(0.12)$ & $8.2(0.5)$ & 0.2 \\
\hline & $4_{2,2}-3_{1,2}$ & 218.440 & 45.5 & $-4.1(0.1)$ & $3.01(0.17)$ & $7.3(0.5)$ & 0.2 \\
\hline & $21_{1,20}-21_{0,21}$ & 227.095 & 557.1 & $-3.7(0.4)$ & $0.87(0.06)$ & $9.3(0.9)$ & 0.1 \\
\hline & $16_{1,16}-15_{2,13}$ & 227.815 & 327.2 & $-4.0(0.2)$ & $1.62(0.07)$ & $8.2(0.4)$ & 0.1 \\
\hline
\end{tabular}



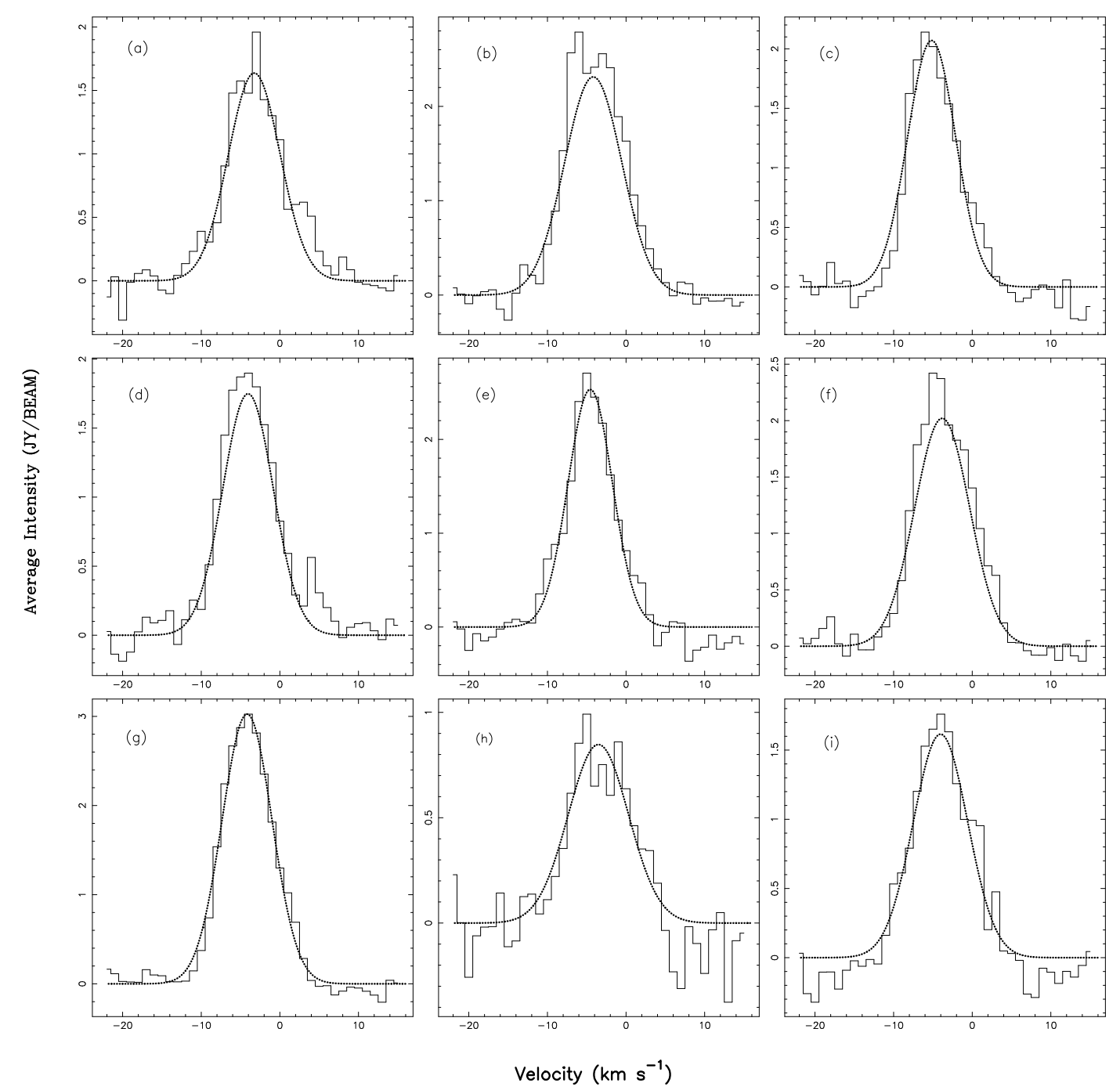

Fig. 3.- Molecular spectra at the peak position of different molecular core. The solid and dashed curves are the observed spectra and the Gaussian fitting to the spectra, respectively.

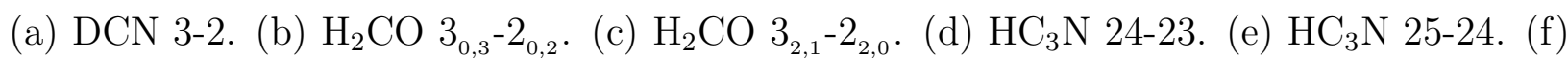
$\mathrm{CH}_{3} \mathrm{OH} 5_{1,4}-4_{2,2}$. (g) $\mathrm{CH}_{3} \mathrm{OH} 4_{2,2}-3_{1,2}$. (h) $\mathrm{CH}_{3} \mathrm{OH} 21_{1,20}-21_{0,21}$. (i) $\mathrm{CH}_{3} \mathrm{OH} 16_{1,16}-15_{2,13}$. 


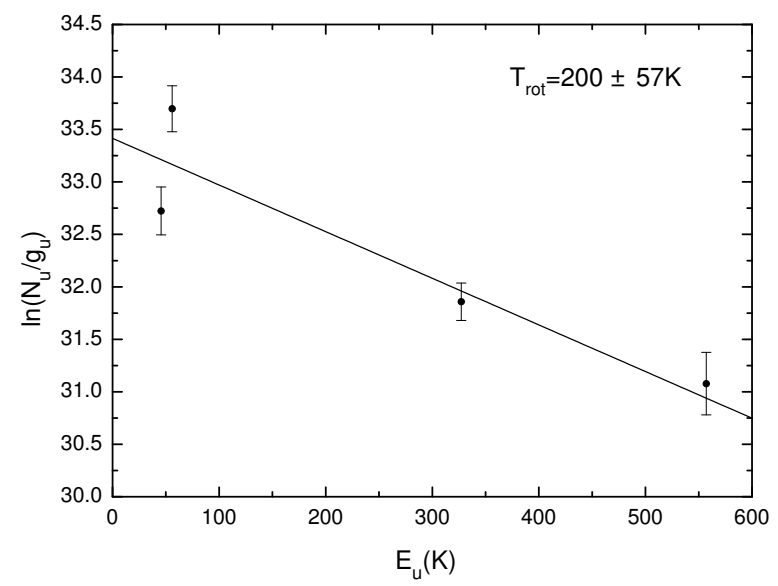

Fig. 4.- Population temperature diagram of the observed $\mathrm{CH}_{3} \mathrm{OH}$ transitions. The vertical bars mark the $\ln \left(N_{\mathrm{u}} / g_{\mathrm{u}}\right)$ errors from the integrated intensities. The linear least-squares fit (solid line) gives a rotation temperature of $200 \pm 57 \mathrm{~K}$. 


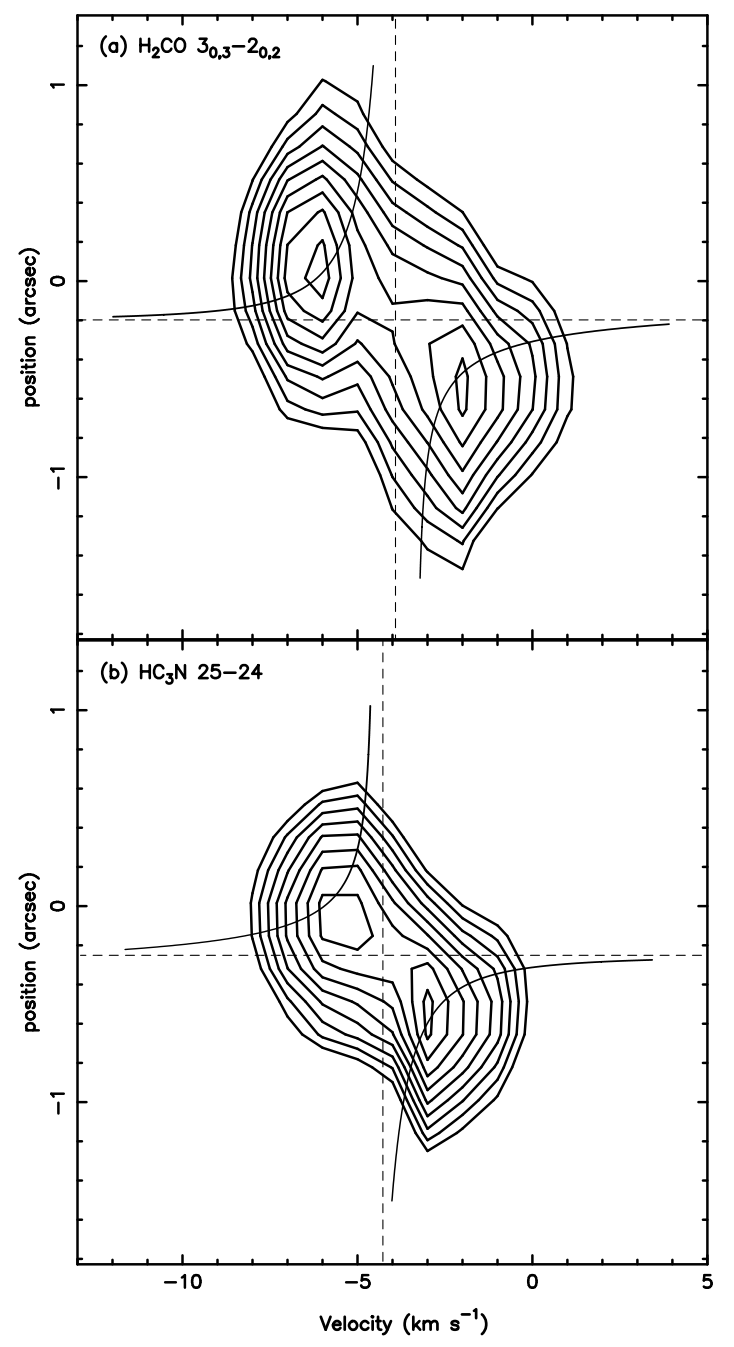

Fig. 5.- (a) The position-velocity plot of the $\mathrm{H}_{2} \mathrm{CO} 3_{0,3}-2_{0,2}$ emission at a position angle of $58^{\circ}$ along the major axis of the disk (Zhang et al. 1999). The contour levels in observed data are plotted at every $2 \sigma .1 \sigma=0.6 \mathrm{Jy}$. Position offsets is $\left(0.6^{\prime \prime}, 1.0^{\prime \prime}\right)$. (b) The position-velocity plot of the $\mathrm{HC}_{3} \mathrm{~N} 24-23$ emission at a position angle of $58^{\circ}$ along the major axis of the disk. The contour levels in observed data are plotted at every $2 \sigma .1 \sigma=0.5 \mathrm{Jy}$. Position offsets is $\left(0.6^{\prime \prime}, 1.2^{\prime \prime}\right)$. The horizontal dashed line marks the center of the disk, and the vertical dashed line marks the cloud systemic velocity. The full lines show Keplerian rotation curve. 